This article may be downloaded for personal use only. Any other use requires prior permission of the author and AIP Publishing. This article appeared in Sun, W.; Davis, R. L.; Thorwirth, S.; Harding, M. E.; van Wijngaarden, J. J. Chem. Phys. 149, 104304 (2018) and may be found at https://doiorg.uml.idm.oclc.org/10.1063/1.5048411

\title{
A highly flexible molecule: The peculiar case of ethynyl isothiocyanate HCCNCS
}

Wenhao Sun, ${ }^{\dagger}$ Rebecca L. Davis, ${ }^{\dagger}$ Sven Thorwirth, ${ }^{\dagger}$ Michael E. Harding, $\|$ and Jennifer van Wijngaarden*广

$\dagger$ Department of Chemistry, University of Manitoba, Winnipeg, Manitoba, R3T 2N2, Canada + I. Physikalisches Institut, Universität zu Köln, Zülpicher Str. 77, 50937 Köln, Germany

"Institut für Nanotechnologie, Karlsruher Institut für Technologie (KIT), Campus Nord, Postfach 3640, 76021 Karlsruhe, Germany

*Corresponding author

Email: vanwijng@cc.umanitoba.ca

Phone: (204)474-8379

Fax: (204)474-7608 


\begin{abstract}
The rotational spectrum of the parent isotopic species of HCCNCS, along with those of three ${ }^{13} \mathrm{C}$ singly substituted variants and one ${ }^{34} \mathrm{~S}$ minor isotopologue, has been observed with high resolution using Fourier transform microwave (FTMW) spectroscopy from 4-26 GHz. Based on the observed spectral pattern and assignment of ${ }^{14} \mathrm{~N}$ nuclear quadrupole hyperfine structure, the geometry of HCCNCS is effectively linear which is further supported by the observation of $\boldsymbol{\ell}$-type doubling for rotational transitions in an excited bending state of the parent HCCNCS. The experimental observations are supported by high level quantum chemical calculations using the coupled-cluster singles and doubles model augmented by a perturbative correction for triple excitations, $\operatorname{CCSD}(\mathrm{T})$, level of theory that reveal an extremely shallow potential energy well along the CNC bending angle with a surprising basis set dependence.
\end{abstract}




\section{$\underline{\text { I. Introduction }}$}

Small molecules containing isocyanate (NCO) or isothiocyanate (NCS) functional groups as well as selected structural isomers (such as $\mathrm{CH}_{3} \mathrm{NCO},{ }^{1} \mathrm{HNCO},{ }^{2} \mathrm{HOCN},{ }^{3,4} \mathrm{HCNO},{ }^{5} \mathrm{HNCS},{ }^{6}$ $\mathrm{HSCN}^{7}$ ) have been detected by astronomers in molecular clouds based on their rotational transitions. The identification of multiple isomers, in particular, provides key input for modelling the chemistry of the interstellar medium (ISM) as the observed isomeric abundances are critically dependent on the underlying formation pathway of each species. ${ }^{8}$ The four isomers of isocyanic acid (HNCO), for example, are predicted to have relative energies of: $0 \mathrm{kcal} \mathrm{mol}^{-1}$ (HNCO), $24.7 \mathrm{kcal} \mathrm{mol}^{-1}$ (HOCN), $70.7 \mathrm{kcal} \mathrm{mol}^{-1}$ (HCNO) and $84.1 \mathrm{kcal} \mathrm{mol}^{-1}$ (HONC), respectively using coupled cluster methods ${ }^{3}$ and while the three lowest energy forms have been detected in the ISM, their relative abundances differ in various regions and do not follow equilibrium thermodynamic predictions. For example, although they differ in energy by $46 \mathrm{kcal}$ $\mathrm{mol}^{-1}$, the abundance ratio of $\mathrm{HCNO} / \mathrm{HOCN}$ is nearly unity in cold sources but the relative abundance of the higher energy isomer HCNO drops as temperature increases. ${ }^{8}$ In surveys toward warmer sources such as $\mathrm{Sgr}$ B2, $\mathrm{HCNO}$ is not detected at all. ${ }^{9}$

The isovalent S-containing analogs have also been explored in terms of the influence of the larger, less electronegative atom on the structure and energetics of the isomers. The four isomers of isothiocyanic acid (HNCS) follow the same energy ordering as in HNCO but cover roughly half the range with relative energies of $0.0 \mathrm{kcal} \mathrm{mol}^{-1}(\mathrm{HNCS}), 6.6 \mathrm{kcal} \mathrm{mol}^{-1}(\mathrm{HSCN})$, $35.1 \mathrm{kcal} \mathrm{mol}^{-1}$ (HCNS) and $36.6 \mathrm{kcal} \mathrm{mol}^{-1}$ (HSNC) from ae-CCSD(T)/cc-pwCVQZ calculations with zero point energy corrections. ${ }^{10}$ The lowest energy form (HNCS) was first studied using waveguide-based microwave spectroscopy by Beard and Dailey ${ }^{11}$ while the second most stable isomer (HSCN) was observed more than 50 years later via FTMW spectroscopy by 
Brünken et.al. ${ }^{12}$ Both forms have been observed in Sgr B2 and TMC-1 with HNCS/HSCN abundance ratios of $\sim 3$ and $\sim 1.4$ in the two sources, respectively ${ }^{7,13}$ and it has been suggested that they form via a common precursor $\mathrm{HNCSH}^{+} .{ }^{7}$ In $2016, \mathrm{McGuire}$ et al ${ }^{10}$ were the first to report rotational spectra of the two remaining isomers, HCNS and HSNC, but to date, these have not been identified in the ISM from examining data taken toward Sgr B2(N). The recent astronomical detection of the HCS/HSC pair of isomers in the dense cloud L483 with greater abundance than $\mathrm{HCO}$ provides increased impetus to improve models of sulfur chemistry in astrophysical environments. $^{14}$

Based on the 200 known interstellar molecules (see, e.g. the Cologne Database for Molecular Spectroscopy), ${ }^{15}$ it is clear that certain functional groups encourage formation of carbon chain molecules in the ISM with polyyne or cumulene backbones. The interstellar detection of a series of $\mathrm{CN}$-containing carbon chains (cyanopolyynes), $\mathrm{H}(\mathrm{C} \equiv \mathrm{C})_{\mathrm{n}} \mathrm{CN}(\mathrm{n}=0 \ldots 4)$, in Heiles's cloud 2 is a good example ${ }^{16-19}$ with $\mathrm{H}(\mathrm{C} \equiv \mathrm{C})_{4} \mathrm{CN}$ being the longest linear species identified in the ISM to date. ${ }^{20}$ Sulfur, on the other hand, has a tendency to seed cumulenic chains of type: $\mathrm{H}_{2} \mathrm{C}_{\mathrm{n}} \mathrm{S}, \mathrm{HC}_{\mathrm{n}} \mathrm{S}^{21}$ and $\mathrm{C}_{\mathrm{n}} \mathrm{S},{ }^{22,23}$ a number of which have been well-characterized in the laboratory as summarized by McCarthy and Thaddeus. ${ }^{24}$ With the exception of $\mathrm{C}_{2} \mathrm{~S},{ }^{25}$ $\mathrm{C}_{3} \mathrm{~S}^{26,27}$ and $\mathrm{C}_{5} \mathrm{~S}^{27,28}$ no $\mathrm{S}$-bearing carbon chains $(\mathrm{n}>1)$ have been identified in space. As the isomers of HNCS provide an interesting range of geometries from linear (HCNS) to bent $\left(\angle \mathrm{HNC}=131^{\circ}\right.$ in $\mathrm{HNCS}, \angle \mathrm{HSC}=94.3^{\circ}$ in $\mathrm{HSCN}, \angle \mathrm{HSN}=95.4^{\circ}$ in $\left.\mathrm{HSNC}\right),{ }^{10}$ the investigation of carbon chains carrying the -NCS group affords the opportunity to explore the geometry of related molecules using microwave spectroscopy and quantum chemical calculations.

Select isocyanates and isothiocyanates related to HCCNCS have already been characterized by their pure rotational spectra. The microwave spectrum of the oxygen analog 
HCCNCO, prepared via pyrolysis, was reported by Ross et al. ${ }^{29}$ and assigned to rotational transitions in the ground and excited CNC bending states. Together with its isoelectronic analogue, $\mathrm{NCNCO},{ }^{30}$ these molecules are prototypical examples of quantum monodromy as the shapes of the underlying potential energy surfaces along the $\mathrm{CNC}$ angle resemble the bottom of a champagne bottle. Based on their reported microwave spectra and analysis using a generalized semi-rigid bender model, the equilibrium structures are bent at nitrogen $\left(129^{\circ}\right.$ in $\mathrm{NCNCO}$ and $140^{\circ}$ in $\left.\mathrm{HCCNCO}\right)$ and the barrier to linearity is nearly halved when $\mathrm{HCC}\left(537.2 \mathrm{~cm}^{-1}\right)$ replaces NC $\left(1014.8 \mathrm{~cm}^{-1}\right) .{ }^{31}$ With S-substitution to form NCNCS, the geometry at nitrogen is less bent $\left(143^{\circ}\right)$ and the monodromy point is nearly halved again $\left(270 \mathrm{~cm}^{-1}\right)$ giving rise to complex vibration-rotation energy level patterns which have been extensively studied in the microwave through infrared regions. ${ }^{32-35}$ This raises a question regarding the extension to the HCCNCS molecule which might be expected to exhibit an even smaller energy difference between the linear and bent arrangement. To the best of our knowledge, HCCNCS has not been investigated in any spectral region before now.

In this article, the first spectroscopic observation of HCCNCS is reported. The title species was produced via electric discharge of a gas mixture outlined below and its rotational spectrum consists of transitions assigned to the parent species as well as those attributed to the three ${ }^{13} \mathrm{C}$ singly substituted species and one ${ }^{34} \mathrm{~S}$ substituted isotopologue. Furthermore, rotational transitions within a degenerate bending mode were also observed. To further understand the nature of the potential energy surface of this highly fluxional molecule, calculations with the coupled-cluster singles and doubles model augmented by a perturbative correction for triple excitations, $\operatorname{CCSD}(\mathrm{T})$, level of theory ${ }^{36}$ with various basis sets were conducted. 


\section{Experimental Methods}

The rotational spectrum of HCCNCS was initially recorded in $2 \mathrm{GHz}$ segments using the chirped pulse Fourier transform (cp-FTMW) microwave spectrometer $(8-18 \mathrm{GHz})$ at the University of Manitoba. ${ }^{37}$ Following tentative assignment of the most intense transitions of HCCNCS, these same features were re-investigated with higher resolution with the Balle-Flygare FTMW spectrometer ${ }^{38}$ to resolve the ${ }^{14} \mathrm{~N}$ quadrupole hyperfine splitting. Spectral linewidths were typically $\sim 7 \mathrm{kHz}$ (FWHM) and lines centers determined within $\pm 2 \mathrm{kHz}$. As the cavity instrument offers greater sensitivity, the spectra of the ${ }^{34} \mathrm{~S}$ and ${ }^{13} \mathrm{C}$ minor isotopologues were then recorded in natural abundance by step-scanning.

Both spectrometers are equipped with a discharge nozzle assembly as shown in Figure 1 affixed to the nozzle. Within the acetal Delrin cap, two copper ring electrodes ( $5 \mathrm{~mm}$ thick) are separated by an insulating spacer ( $8 \mathrm{~mm}$ thick). A second spacer ( $5 \mathrm{~mm}$ thick) isolates the electrodes from the nozzle flange. All components seated together leave a $2.5 \mathrm{~mm}$ diameter channel from the nozzle orifice ( $1 \mathrm{~mm}$ diameter) to the vacuum chamber. A continuous negative voltage of $650 \mathrm{~V}-950 \mathrm{~V}$ was applied to the electrode closest to the nozzle while the second was grounded. The discharge can be observed as a flashing glow as it is modulated by the $7 \mathrm{~Hz}$ repetition rate of the pulsed nozzle. 
Figure 1. Schematic diagram of the electric discharge assembly made of acetal Delrin which couples to a General Valve (Series 9) nozzle.

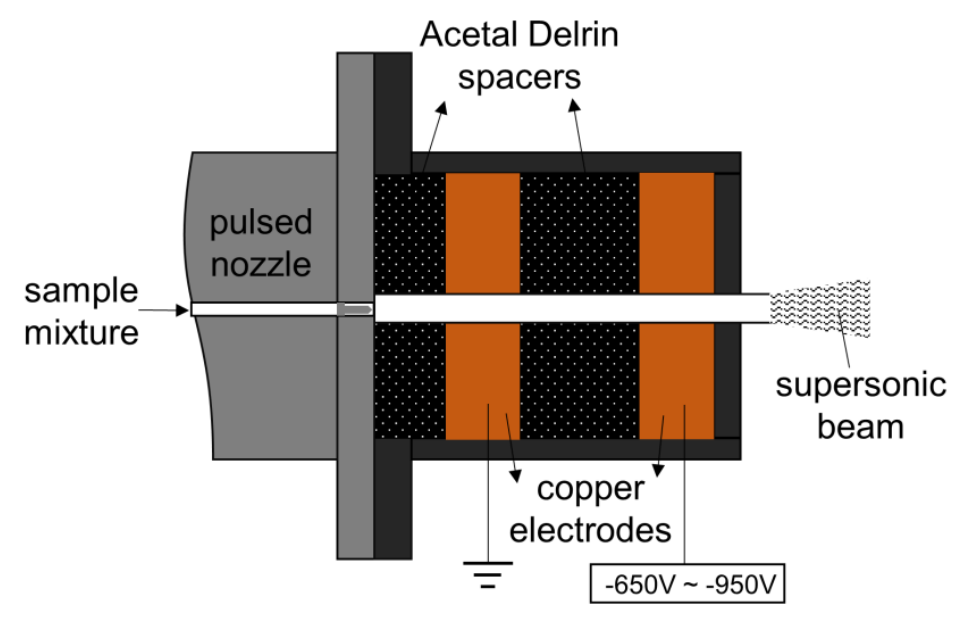

The title compound was produced in a supersonic jet expansion of the discharge products from a gas mixture containing $\mathrm{CH}_{3} \mathrm{NCS}$ (Sigma Aldrich Canada, 97\%). As $\mathrm{CH}_{3} \mathrm{NCS}$ is solid at room temperature (mp: $30-34^{\circ} \mathrm{C}$ ), a small volume was heated in a glass bubbler seated in a bath maintained at $37^{\circ} \mathrm{C}$. A gas mixture of acetylene (1\%) in neon (1.5 atm) was bubbled through 2 $\mathrm{mL}$ of liquid $\mathrm{CH}_{3} \mathrm{NCS}$ before passing to the discharge nozzle. To identify discharge dependent species, spectra were recorded with both the discharge voltage turned on and off to allow removal of transitions from the $\mathrm{CH}_{3} \mathrm{NCS}$ precursor.

\section{Computational Methods}

Preliminary B3LYP ${ }^{39-41}$ and valence-only MP2 ${ }^{42}$ calculations using aug-cc-pVTZ ${ }^{43-45}$ and cc-pVQZ ${ }^{43,45}$ basis sets were performed employing the Gaussian 09 package. ${ }^{46}$ As these first calculations produced both linear and nonlinear equilibrium geometries depending on the level of theory and basis set employed, subsequent quantum chemical calculations were performed with the CFOUR (Coupled-Cluster techniques for Computational Chemistry) program. ${ }^{47}$ Although 
second-order vibrational perturbation theory (VPT2) $)^{48}$ has conceptual difficulties with the description of large amplitude motions (and with vibrationally floppy molecules in general), VPT2 was used in a preliminary approach to compute the rotational constants and the corresponding vibrational corrections. Quadratic, cubic, and semi-diagonal quartic force constants as required for the application of VPT2 were computed via numerical differentiation of analytic second derivatives at the coupled-cluster singles and doubles level extended by a perturbative correction for the contribution from triple excitations $(\operatorname{CCSD}(\mathrm{T})) .{ }^{49,50} \mathrm{ANO}$, ANO1, ANO2, cc-pV(T+d)Z, cc-pCVTZ and cc-pwCVTZ, cc-pCVXZ, cc-pwCVXZ (X=T, Q, and 5) and aug-cc-pwCVTZ basis sets were employed for the computations using CFOUR. ${ }^{43-}$ $45,51-54$

\section{Spectral Assignment and Fitting}

Based on the initial MP2 and B3LYP computations using aug-cc-pVTZ and cc-pVQZ basis sets, it was assumed that the ground state rotational spectrum of HCCNCS would be that of a linear or quasilinear molecule. Although the MP2 calculations yielded a slightly bent equilibrium structure $\left(\angle \mathrm{CNC}=172.5^{\circ}\right)$ about nitrogen, the predicted rotational constants gave rise to a spectrum similar to that of the linear DFT result as only the $\mathrm{K}_{\mathrm{a}}=0$ levels would be appreciably populated in the jet. Furthermore, the barrier to linearity is negligible at the MP2 level (about $2 \mathrm{~cm}^{-1}$ ) and falls well below the zero point energy of the lowest vibration (about 38 $\mathrm{cm}^{-1}$ at the valence-only MP2 level).

As the first discharge experiments were performed using a broadband cp-FTMW instrument, hundreds of transitions were observed despite the fact that only four transitions due to the parent HCCNCS fall within the instrument range of 8-18 GHz. A number of discharge- 
dependent species were readily identified based on their previously reported microwave spectra. These known species include, for example: $\mathrm{HC}_{3} \mathrm{~N},{ }^{55,56} \mathrm{HC}_{5} \mathrm{~N},{ }^{57} \mathrm{CH}_{3} \mathrm{CN},{ }^{58} \mathrm{NCNCS},{ }^{32} \mathrm{HNCS},{ }^{1,59}$ HSCN. ${ }^{12}$ Within the dense spectrum, regularly-spaced transitions consistent with HCCNCS were identified; a subset of which are shown in Figure 2.

Figure 2. cp-FTMW spectrum obtained from a discharge mixture of $\mathrm{CH}_{3} \mathrm{NCS}$ and $\mathrm{HCCH}$. The top trace is the experimental spectrum from averaging 1.5 million FIDs following removal of precursor lines. The bottom trace is the simulated spectra at $5 \mathrm{~K}$ for HCCNCS and other species identified as discharge products. The simulated intensities are based on dipole moments but do not account for abundance of all discharge products.

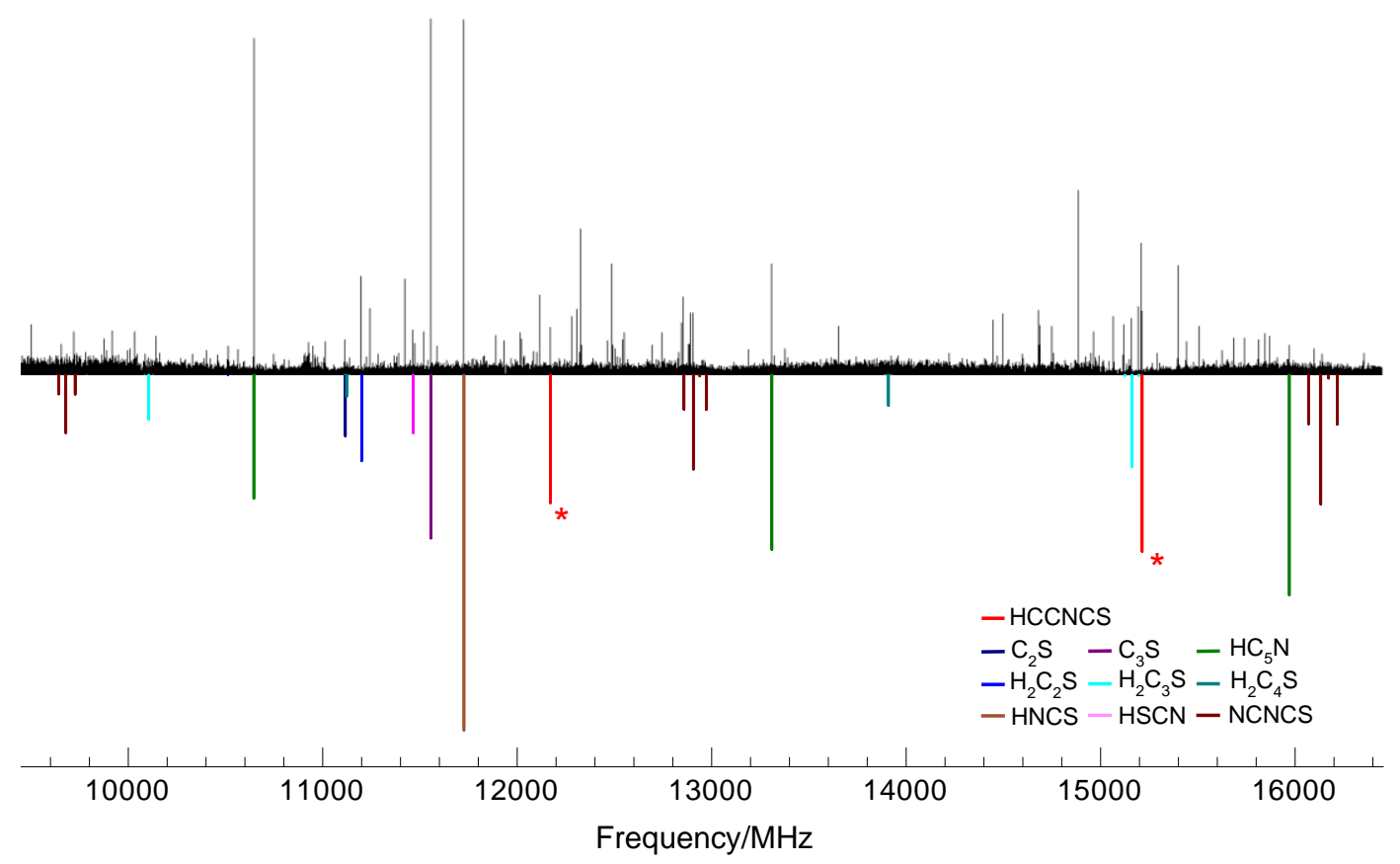


The rotational spectrum of HCCNCS was then re-investigated with the more sensitive, Balle-Flygare instrument to resolve the hyperfine splitting due to the ${ }^{14} \mathrm{~N}$ quadrupole moment and extend the spectral range (4-26 GHz); sample spectra are shown in Figure 3. The assignment was further supported by the detection of transitions from the ${ }^{34} \mathrm{~S}$ analogue and all three ${ }^{13} \mathrm{C}$ isotopologues in natural abundance. The assigned lines are summarized as supplementary material (Tables S1-S5). The rotational spectra of the five isotopologues were fit separately using a linear molecule Hamiltonian in the SPFIT program $;{ }^{60}$ the resulting parameters are presented in Table 1.

Figure $3 .{ }^{14} \mathrm{~N}$ hyperfine structure of $J^{\prime}-J{ }^{\prime \prime}=6-5$ transition from the parent (left), ${ }^{34} \mathrm{~S}$ species (middle) and one ${ }^{13} \mathrm{C}$ species (right) of HCCNCS, collected with 200, 2000 and 32000 cycles, respectively.
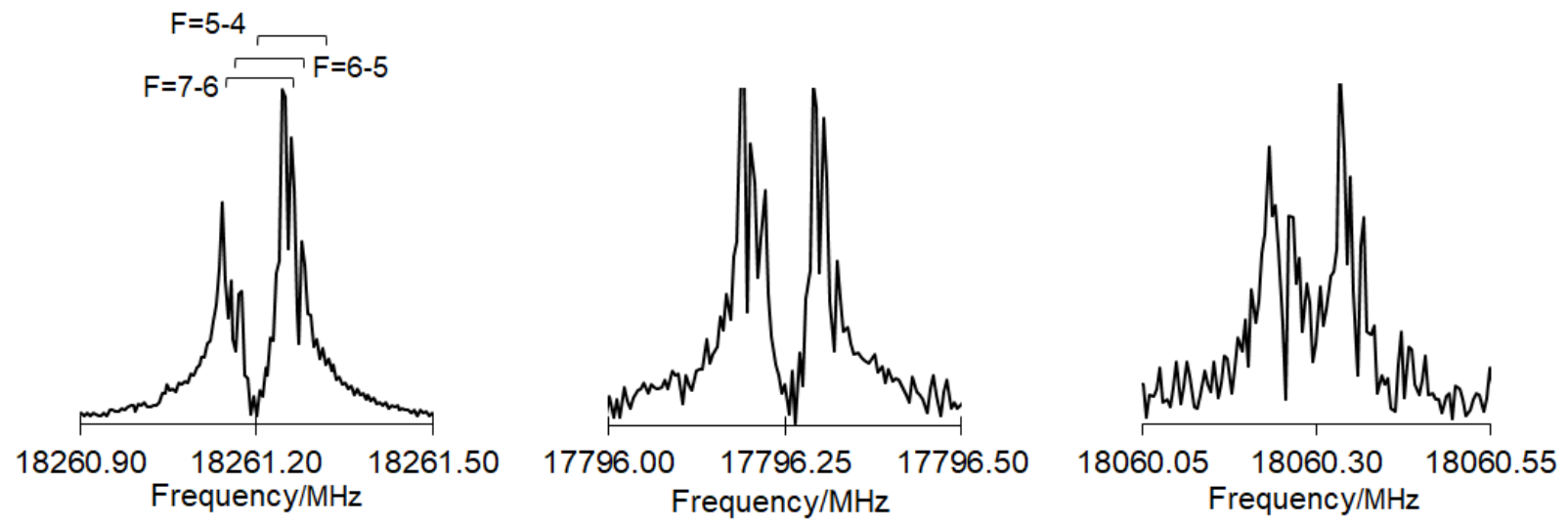
Table 1. Ground State Spectroscopic Constants of the Parent Species of HCCNCS, and Its ${ }^{13} \mathrm{C}$ and ${ }^{34} \mathrm{~S}$ Isotopologues.

\begin{tabular}{|c|c|c|c|c|}
\hline & parent & \multicolumn{2}{|c|}{ CCSD(T)/cc-pwCVTZ (calc.) } & \\
\hline$B / \mathrm{MHz}$ & $1521.77406(4)$ & 1525.0 & & \\
\hline$D / \mathrm{kHz}$ & $0.09630(5)$ & 0.039 & & \\
\hline$e Q q / \mathrm{MHz}$ & $2.360(8)$ & 2.39 & & \\
\hline $\mathrm{rms} / \mathrm{kHz}$ & 1.95 & 1 & & \\
\hline$\mu / \mathrm{D}$ & & 1.79 & & \\
\hline \multirow[t]{2}{*}{ line \# } & 30 & 1 & & \\
\hline & $\mathrm{H}^{13} \mathrm{CCNCS}$ & $\mathrm{HC}^{13} \mathrm{CNCS}$ & $\mathrm{HCCN}^{13} \mathrm{CS}$ & $\mathrm{HCCNC}^{34} \mathrm{~S}$ \\
\hline$B / \mathrm{MHz}$ & $1478.35699(23)$ & $1505.0314(5)$ & $1520.44966(18)$ & $1483.02745(10)$ \\
\hline$D / \mathrm{kHz}$ & $0.0922(24)$ & $0.096(5)$ & $0.0957(18)$ & $0.0899(12)$ \\
\hline$e Q q / \mathrm{MHz}$ & $2.59(22)$ & $2.53(26)$ & $2.27(9)$ & $2.36(3)$ \\
\hline $\mathrm{rms} / \mathrm{kHz}$ & 2.01 & 1.63 & 1.74 & 1.66 \\
\hline line \# & 7 & 5 & 8 & 16 \\
\hline
\end{tabular}

Additional transitions attributed to rotational transitions in an excited vibrational state of the parent HCCNCS were subsequently found via step-scanning the Balle-Flygare instrument. These transitions are split due to $\ell$-type doubling, as shown in Figure 4, and the relative intensity of the $J^{\prime}-J^{\prime \prime}=6-5$ transition was $\sim 5 \%$ that of the ground state. The excited state constants obtained from fitting the transitions are summarized in Table 2; the assigned transitions are provided in the supplementary material (Tables S6, S7). 
Figure 4. The assignment of $\ell$-type doubling and ${ }^{14} \mathrm{~N}$ hyperfine structure of the $J^{\prime}-J{ }^{\prime \prime}=6-5$ transition in an excited vibrational state of HCCNCS.

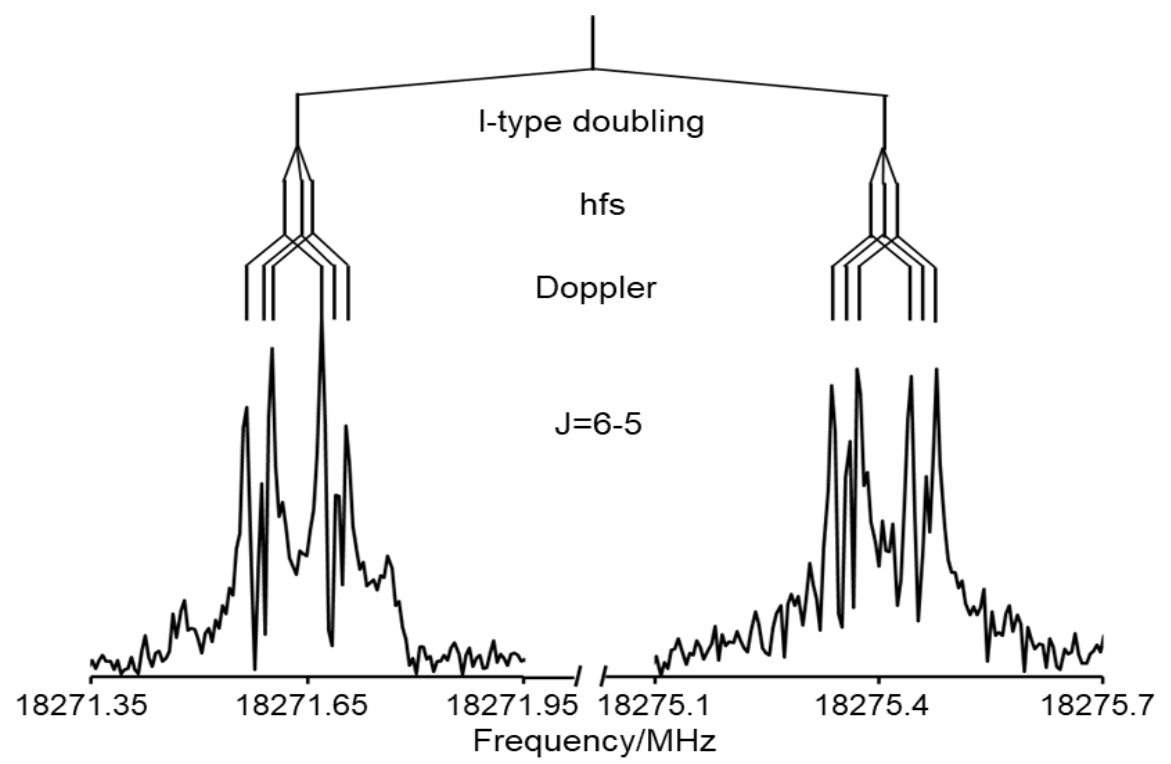

Table 2. Spectroscopic Constants for the Observed Excited Vibrational State of the Parent HCCNCS Species.

\begin{tabular}{cc}
\hline \hline & $\mathrm{v}=1$ \\
\hline$B / \mathrm{MHz}$ & $1522.79930(8)$ \\
$D / \mathrm{kHz}$ & $0.0858(9)$ \\
$e Q q / \mathrm{MHz}$ & $2.34(4)$ \\
$q_{\mathrm{v}} / \mathrm{kHz}$ & $314.21(16)$ \\
$q_{\mathrm{v}} \mathrm{J} / \mathrm{kHz}$ & $0.0034(17)$ \\
$\mathrm{rms} / \mathrm{kHz}$ & 1.58 \\
line \# & 26 \\
\hline \hline
\end{tabular}




\section{Results and Discussion}

Establishing the equilibrium configuration of HCCNCS from calculations is a very challenging endeavour. The structural parameters for HCCNCS obtained from the CCSD(T) calculations are summarized in the supplementary material (Table S8) and show a striking variation with basis set. Nonlinear equilibrium geometries are obtained from valence-only CCSD(T) computations employing ANO0, ANO1, ANO2, cc-pV(T+d)Z, cc-pCVTZ and ccpwCVTZ. All electron CCSD(T) structure optimizations yield linear structures if cc-pCVXZ or cc-pwCVXZ (X=T, Q, and 5) basis sets are used, however, a nonlinear equilibrium structure is found if diffuse functions are added (CCSD(T)/aug-cc-pwCVTZ). It thus appears that CCSD(T) computations employing the aforementioned rather sizable basis sets might not be accurate enough to resolve the question of the equilibrium structure of HCCNCS. This may be due to intramolecular basis set superposition errors as, for example, in the case of the mono-substituted benzenes ${ }^{61}$ or problems arising from the concept of the equilibrium structure itself (see, for example, the discussion in reference 62).

Despite its peculiar nature, as inferred from the difficulties in establishing an equilibrium geometry, the detection of five isotopic species leaves little doubt to the assignment to HCCNCS as being the molecular carrier of the new microwave lines reported here. Effectively, HCCNCS appears linear in the ground vibrational state and the rotational data can be fitted with a simple linear molecule Hamiltonian with hyperfine structure from one quadrupolar nucleus. The calculated rotational parameters obtained at the CCSD(T)/cc-pwCVTZ level of theory (yielding a linear equilibrium geometry) are in good agreement with experiment for all isotopologues with the exception of the centrifugal distortion constant which is 2.39 times greater than the calculated value. In a series of papers, Botschwina and co-workers have linked such discrepancies to the 
floppiness of the molecule and correlated the ratio of $D_{0}(\mathrm{expt}) / D_{e}$ (theory) to the flatness of the bending potential of the chain by comparing results from series of astronomically relevant molecules of type $\mathrm{C}_{2 \mathrm{n}+1} \mathrm{O}, \mathrm{C}_{2 \mathrm{n}+1} \mathrm{~N}^{-}, \mathrm{C}_{2 \mathrm{n}} \mathrm{H}^{-}, \mathrm{NC}_{2 \mathrm{n}-1} \mathrm{H}$ among others. ${ }^{63,64}$ Upon comparison with known species, the ratio of $D_{0}(\operatorname{expt}) / D_{e}$ (theory) of HCCNCS (2.39) derived in the present study is intermediate to that of highly flexible $\mathrm{C}_{3}(7.26)$ and $l-\mathrm{C}_{3} \mathrm{H}^{+}(1.80)^{65}$ lending support our assertion that this molecule is incredibly floppy.

To better understand this flexibility and the nature of the potential along the CNC bending coordinate of HCCNCS in comparison with its isoelectronic (NCNCS) and isovalent HCCNCO, NCNCO) counterparts, the energy dependence as a function of $\rho$ (the deviation from linearity at nitrogen) was computed at the fc-CCSD(T)/cc-pV(T+d)Z level. The results are presented in Figure 5 (top). The trend that the equilibrium geometry of the molecule becomes less bent (with a lower barrier to linearity) when moving from NCNCO to HCCNCO is also evident for the S-analogues with HCCNCS appearing almost linear on the scale shown. The basis set dependence of the CNC bending potential of HCCNCS was explored with further $\operatorname{CCSD}(\mathrm{T})$ calculations and the results presented in Figure 5 (bottom). Given the subtle energy difference (of just a few $\mathrm{cm}^{-1}$ ) between linear and nonlinear structures, as well as the very small change in energy (again just a few $\mathrm{cm}^{-1}$ ) observed along the bending coordinate in Figure 5 (bottom), it seems mandatory that higher level correlation effects beyond CCSD(T), relativistic effects and Born-Oppenheimer corrections together with large basis set expansions be considered in the future. Also, extension beyond the straightforward VPT2 picture necessitates the development of a highly accurate full dimensional potential energy surface. These theoretical considerations are beyond the scope of this study and the present analysis is based on the fact 
that the experimentally observed rotational features belong to an effectively linear species due to the symmetry of the bending motion.

Figure 5: Energy dependence as a function of the $\mathrm{CNC}$ bending angle $\rho$ : Top- At the fc$\operatorname{CCSD}(\mathrm{T}) / \mathrm{cc}-\mathrm{pV}(\mathrm{T}+\mathrm{d}) \mathrm{Z}$ level of theory for HCCNCS and isoelectronic and isovalent species; Bottom- At the CCSD(T) level of theory employing cc-pV(T+d)Z, cc-pwCVTZ, and aug-ccpwCVTZ basis sets for HCCNCS.

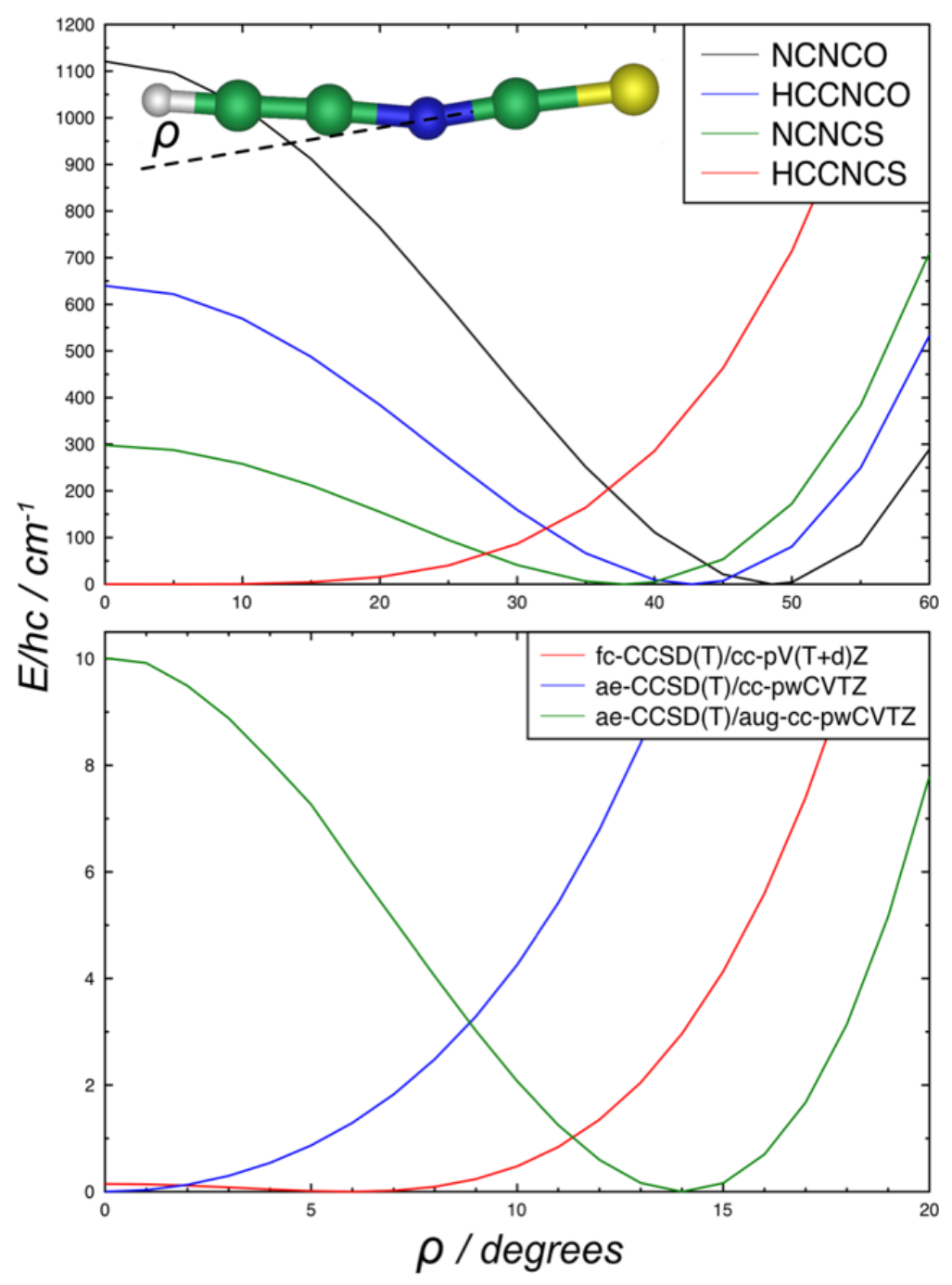


Assuming linearity, the bond lengths of HCCNCS were estimated via least squares fitting of the equilibrium rotational constants, $B_{e}$, of the five isotopologues (obtained by combining vibrational corrections computed at the VPT2 CCSD(T)/cc-pwCVTZ level of theory and the experimental rotational constants of the isotopologues) as input to Kisiel's STRFIT program. ${ }^{66}$ The results, presented in Table 3, are in excellent agreement with the equilibrium structure computed at the CCSD(T) level. Although all bond lengths become slightly shorter with increasing basis set size, all results are consistent with the semi-empirical structure.

At present, the putative vibrational satellites from a first excited degenerate bending vibrational mode cannot be assigned to any particular vibrational state with confidence owing to the rather large scatter in the vibration-rotation interaction constants calculated using various basis sets. From a more general trend in the calculations, it seems likely, however, that the lines do not originate from the energetically lowest-lying CNC-bending mode. Such a finding would not be uncommon and would be supported by results from earlier (FTMW) studies, which showed that low-lying vibrational modes may be cooled efficiently or frozen out under the conditions of a free jet expansion such as the one used here. ${ }^{67}$ 
Table 3. Equilibrium bond lengths $\left(r_{e}\right)$ at the $\operatorname{CCSD}(T)$ level of theory and semi-experimental bond lengths $\left(\mathrm{r}_{\mathrm{e}}^{\mathrm{SE}}\right)$ from least squares fitting in $\AA$.

\begin{tabular}{ccccc}
\hline \hline & $\mathrm{r}_{\mathrm{e}}{ }^{\mathrm{SE}}$ & $\mathrm{r}_{\mathrm{e}}($ cc-pwCVTZ) & $\mathrm{r}_{\mathrm{e}}$ (cc-pwCVQZ) & $\mathrm{r}_{\mathrm{e}}$ (cc-pwCV5Z) \\
\hline $\mathrm{R}(\mathrm{H} 1 \mathrm{C} 2)$ & $1.0621^{1}$ & 1.0621 & 1.0612 & 1.0610 \\
$\mathrm{R}(\mathrm{C} 2 \mathrm{C} 3)$ & $1.207(1)$ & 1.2093 & 1.2070 & 1.2063 \\
$\mathrm{R}(\mathrm{C} 3 \mathrm{~N} 4)$ & $1.304(3)$ & 1.3009 & 1.2991 & 1.2988 \\
$\mathrm{R}(\mathrm{N} 4 \mathrm{C} 5)$ & $1.192(5)$ & 1.1888 & 1.1870 & 1.1866 \\
$\mathrm{R}(\mathrm{C} 5 \mathrm{~S} 6)$ & $1.573(3)$ & 1.5726 & 1.5698 & 1.5690 \\
\hline \hline
\end{tabular}

${ }^{1}$ For the fit, the $\mathrm{H}-\mathrm{C}$ bond length was fixed to $\mathrm{r}_{\mathrm{e}}=1.0621 \AA$ as the deuterated species was not studied.

As HCCNCS comprises of ethynyl- $\left(\mathrm{C}_{2} \mathrm{H}\right)$ and NCS groups, both of which are structural elements of known and abundant astronomical molecules, it might itself be an interesting target for future radio astronomical searches. The spectroscopic data collected here permit such deep searches to be carried out in the centimeter wave range. Laboratory investigation of the spectra of the deuterated and $\mathrm{H}^{13} \mathrm{C}^{13} \mathrm{CNCS}$ isotopologues produced using enriched samples would provide useful information for geometry determination and potential formation pathways in space, respectively. Future laboratory studies in the millimeter wave range would be beneficial to further constrain centrifugal distortion effects and to permit a more comprehensive study of the vibrational satellite pattern and possibly quasilinearity. It would also be worthwhile to extend this work to the study of structural isomers. Linear HCCCNS, for example, is calculated here to lie only $20.3 \mathrm{kcal} \mathrm{mol}^{-1}$ above HCCNCS in energy and is substantially polar (3.64 D) at the $\mathrm{CCSD}(\mathrm{T}) / \mathrm{cc}-\mathrm{pwCVTZ}$ (no zero-point vibrational corrections) level of theory.

In closing, the rotational spectrum of HCCNCS in its ground and an excited vibrational state has been reported here for the first time. The assignment is confirmed by the observation of 
the ${ }^{34} \mathrm{~S}$ and ${ }^{13} \mathrm{C}$ minor isotopic species. The bond lengths derived for the semi-empirical structure are in excellent agreement with calculations at the $\operatorname{CCSD}(\mathrm{T})$ level, however, it is surprisingly difficult to establish the equilibrium geometry of HCCNCS due to its extremely flat potential. Future theoretical studies including the derivation of a highly accurate potential energy surface would be helpful to model the vibrational-rotational behavior of HCCNCS.

\section{Supplementary Material}

See supplementary material for the lists of assigned transitions and the calculated structural parameters of HCCNCS.

\section{$\underline{\text { Acknowledgements }}$}

Research in the group of J.vW. is funded by the Natural Sciences and Engineering Research Council of Canada (NSERC) through the Discovery Grant program. W.S. is grateful for financial support provided through a University of Manitoba Graduate Fellowship and the GETS program from the Faculty of Graduate Studies. M.E.H. acknowledges support of the Bundesministerium für Bildung und Forschung (BMBF) through the Helmholtz Research Program POF Science and Technology of Nanosystems. S.T. thanks the Deutsche Forschungsgemeinschaft (DFG) for support through grants SFB 956 and SCHL 341/15-1 (Cologne Center for Terahertz Spectroscopy). 


\section{$\underline{\text { References }}$}

${ }^{1}$ D.T. Halfen, V.V. Ilyushin, and L.M. Ziurys, Astrophys. J. Lett. 812, L5 (2015).

${ }^{2}$ L.E. Snyder and D. Buhl, Astrophys. J. 177, 619 (1972).

${ }^{3}$ S. Brünken, C.A. Gottlieb, M.C. McCarthy, and P. Thaddeus, Astrophys. J. 697, 880 (2009).

${ }^{4}$ S. Brünken, A. Belloche, S. Martin, L. Verheyen, and K.M. Menten, Astron. Astrophys. 516, A109 (2010).

${ }^{5}$ N. Marcelino, J. Cernicharo, B. Tercero, and E. Roueff, Astrophys. J. Lett. 690, 27 (2009).

${ }^{6}$ M.A. Frerking, R.A. Linke, and P. Thaddeus, Astrophys. J. 234, L143 (1979).

${ }^{7}$ D.T. Halfen, L.M. Ziurys, S. Brünken, C.A. Gottlieb, M.C. McCarthy, and P. Thaddeus, Astrophys. J. Lett. 702, 124 (2009).

${ }^{8}$ D. Quan, E. Herbst, Y. Osamura, and E. Roueff, Astrophys. J. 725, 2101 (2010).

${ }^{9}$ N. Marcelino, S. Brünken, J. Cernicharo, D. Quan, E. Roueff, E. Herbst, and P. Thaddeus, Astron. Astrophys. 516, A105 (2010).

${ }^{10}$ B.A. McGuire, M.-A. Martin-Drumel, S. Thorwirth, S. Brünken, V. Lattanzi, J.L. Neill, S. Spezzano, Z. Yu, D.P. Zaleski, A.J. Remijan, B.H. Pate, and M.C. McCarthy, Phys. Chem. Chem. Phys. 18, 22693 (2016).

${ }^{11}$ C.I. Beard and B.P. Dailey, J. Chem. Phys. 18, 1437 (1950).

${ }^{12}$ S. Brünken, Z. Yu, C.A. Gottlieb, M.C. McCarthy, and P. Thaddeus, Astrophys. J. 706, 1588 (2009).

${ }^{13}$ G.R. Adande, D.T. Halfen, L.M. Ziurys, D. Quan, and E. Herbst, Astrophys. J. 725, 561 (2010).

${ }^{14}$ M. Agúndez, N. Marcelino, J. Cernicharo, and M. Tafalla, Astron. Astrophys. 611, L1 (2018).

${ }^{15}$ H.S.P. Müller, S. Thorwirth, D.A. Roth, and G. Winnewisser, Astron. Astrophys. 370, L49 
(2001).

${ }^{16}$ L.E. Snyder and D. Buhl, Astrophys. J. 163, 47 (1971).

${ }^{17}$ B.E. Turner, Astrophys. J. 163, 35 (1971).

${ }^{18}$ L.T. Little, G.H. Macdonald, P.W. Riley, and D.N. Matheson, Mon. Not. R. Astron. Soc. 183, 45 (2018).

${ }^{19}$ N.W. Broten, T. Oka, L.W. Avery, J.M. Macleod, and H.W. Kroto, Astrophys. J. 223, 105 (1978).

${ }^{20}$ Previous astronomical detections of $\mathrm{HC}_{11} \mathrm{~N}$ (Bell et al., Astrophys, J. 483, L61 (1997) and references therein) were not confirmed in the most recent studies (Loomis et al., MNRAS 463, 4175 (2016); Cordiner et al., Astrophys. J. 850, 187(2017)).

${ }^{21}$ V.D. Gordon, M.C. McCarthy, A.J. Apponi, and P. Thaddeus, Astrophys. J. Suppl. Ser. 138, 297 (2002)

${ }^{22}$ V.D. Gordon, M.C. McCarthy, A.J. Apponi, and P. Thaddeus, Astrophys. J. Suppl. Ser. 134, $311(2001)$

${ }^{23}$ B.A. McGuire, M.-A. Martin-Drumel, K.L.K. Lee, J.F. Stanton, C.A. Gottlieb, and M.C. McCarthy, Phys. Chem. Chem. Phys. 20, 13870 (2018).

${ }^{24}$ M.C. McCarthy and P. Thaddeus, Chem. Soc. Rev. 30, 177 (2001).

${ }^{25}$ S. Saito, K. Kawaguchi, S. Yamamoto, M. Ohishi, and H. Suzuki, Astrophys. J. 317, L115 (1987).

${ }^{26}$ S. Yamamoto, S. Saito, K. Kawaguchi, N. Kaifu, and H. Suzuki, Astrophys. J. 317, L119 (1987).

${ }^{27}$ M.B. Bell, L.W. Avery, and P.A. Feldman, Astrophys. J. 417, L37 (1993).

${ }^{28}$ M. Agúndez, J. Cernicharo, and M. Guélin, Astron. Astrophys. 570, A45 (2014). 
${ }^{29}$ S.C. Ross, T.A. Cooper, S. Firth, H.W. Kroto, and D.R.M. Walton, J. Mol. Spectrosc. 152, 152 (1992).

${ }^{30}$ W.H. Hocking and M.C.L. Gerry, J. Mol. Spectrosc. 59, 338 (1976).

${ }^{31}$ M. Winnewisser, B.P. Winnewisser, I.R. Medvedev, F.C. De Lucia, S.C. Ross, and L.M. Bates, J. Mol. Struct. 798, 1 (2006).

${ }^{32}$ M.A. King, H.W. Kroto, and B.M. Landsberg, J. Mol. Spectrosc. 113, 1 (1985).

${ }^{33}$ B.P. Winnewisser, M. Winnewisser, I.R. Medvedev, M. Behnke, F.C. De Lucia, S.C. Ross, and J. Koput, Phys. Rev. Lett. 95, 243002 (2005).

${ }^{34}$ B.P. Winnewisser, M. Winnewisser, I.R. Medvedev, F.C. De Lucia, S.C. Ross, and J. Koput, Phys. Chem. Chem. Phys. 12, 8158 (2010).

${ }^{35}$ M. Winnewisser, B.P. Winnewisser, F.C. De Lucia, D.W. Tokaryk, S.C. Ross, and B.E. Billinghurst, Phys. Chem. Chem. Phys. 16, 17373 (2014).

${ }^{36}$ K. Raghavachari, G.W. Trucks, J.A. Pople, and M. Head-Gordon, Chem. Phys. Lett. 157, 479 (1989).

${ }^{37}$ L. Evangelisti, G. Sedo, and J. van Wijngaarden, J. Phys. Chem. A 115, 685 (2011).

${ }^{38}$ G. Sedo and J. van Wijngaarden, J. Chem. Phys. 131, 044303 (2009).

${ }^{39}$ A.D. Becke, Phys. Rev. A 38, 3098 (1988).

${ }^{40}$ A.D. Becke, J. Chem. Phys. 98, 5648 (1993).

${ }^{41}$ P.J. Stephens, F.J. Devlin, C.F. Chabalowski, and M.J. Frisch, J. Phys. Chem. 98, 11623 (1994).

${ }^{42}$ C. Møller and M.S. Plesset, Phys. Rev. 46, 618 (1934).

43 T.H. Dunning, J. Chem. Phys. 90, 1007 (1989).

${ }^{44}$ R.A. Kendall, T.H. Dunning, and R.J. Harrison, J. Chem. Phys. 96, 6796 (1992). 
${ }^{45}$ D.E. Woon and T.H. Dunning, J. Chem. Phys. 98, 1358 (1993).

${ }^{46}$ M.J. Frisch, G.W. Trucks, H.B. Schlegel, G.E. Scuseria, M.A. Robb, J.R. Cheeseman, G. Scalmani, V. Barone, B. Mennucci, G.A. Petersson, H. Nakatsuji, M. Caricato, X. Li, H.P. Hratchian, A.F. Izmaylov, J. Bloino, G. Zheng, J.L. Sonnenberg, M. Hada, M. Ehara, K. Toyota, R. Fukuda, J. Hasegawa, M. Ishida, T. Nakajima, Y. Honda, O. Kitao, H. Nakai, T. Vreven, J.A. Montgomery, J.E. Peralta, F. Ogliaro, M. Bearpark, J.J. Heyd, E. Brothers, K.N. Kudin, V.N. Staroverov, R. Kobayashi, J. Normand, K. Raghavachari, A. Rendell, J.C. Burant, S.S. Iyengar, J. Tomasi, M. Cossi, N. Rega, J.M. Millam, M. Klene, J.E. Knox, J.B. Cross, V. Bakken, C. Adamo, J. Jaramillo, R. Gomperts, R.E. Stratmann, O. Yazyev, A.J. Austin, R. Cammi, C. Pomelli, J.W. Ochterski, R.L. Martin, K. Morokuma, V.G. Zakrzewski, G.A. Voth, P. Salvador, J.J. Dannenberg, S. Dapprich, A.D. Daniels, Farkas, J.B. Foresman, J. V Ortiz, J. Cioslowski, and D.J. Fox, Gaussian 09, Revis. B.01, Gaussian, Inc., Wallingford CT (2010).

${ }^{47}$ CFOUR, a quantum chemical program package written by J.F. Stanton, J. Gauss, L. Cheng, M.E. Harding, D.A. Matthews, P.G. Szalay with contributions from A.A. Auer, R.J. Bartlett, U. Benedikt, C. Berger, D.E. Bernholdt, Y.J. Bomble, O. Christiansen, F. Engel, R. Faber, M. Heckert, O. Heun, M. Hilgenberg, C. Huber, T.-C. Jagau, D. Jonsson, J. Jusélius, T. Kirsch, K. Klein, W.J. Lauderdale, F. Lipparini, T. Metzroth, L.A. Mück, D.P. O'Neill, D.R. Price, E. Prochnow, C. Puzzarini, K. Ruud, F. Schiffmann, W. Schwalbach, C. Simmons, S. Stopkowicz, A. Tajti, J. Vázquez, F. Wang, J.D. Watts and the integral packages MOLECULE (J. Almlöf and P.R. Taylor), PROPS (P.R. Taylor), ABACUS (T. Helgaker, H.J. Aa. Jensen, P. Jørgensen, and J. Olsen), and ECP routines by A. V. Mitin and C. van Wüllen. For the current version, see http://www.cfour.de.

${ }^{48}$ I.M. Mills, in Mol. Spectrosc. (Elsevier, 1972), pp. 115-140. 
${ }^{49}$ J. Gauss and J.F. Stanton, Chem. Phys. Lett. 276, 70 (1997).

${ }^{50}$ J.F. Stanton, C.L. Lopreore, and J. Gauss, J. Chem. Phys. 108, 7190 (1998).

${ }^{51}$ J. Almlöf and P.R. Taylor, J. Chem. Phys. 86, 4070 (1987).

52 T.H. Dunning, K.A. Peterson, and A.K. Wilson, J. Chem. Phys. 114, 9244 (2001).

${ }^{53}$ D.E. Woon and T.H. Dunning, J. Chem. Phys. 103, 4572 (1995).

${ }^{54}$ K.A. Peterson and T.H. Dunning, J. Chem. Phys. 117, 10548 (2002).

${ }^{55}$ R.A. Creswell, G. Winnewisser, and M.C.L. Gerry, J. Mol. Spectrosc.65, 420 (1977).

${ }^{56}$ S. Thorwirth, H.S.P. Müller, and G. Winnewisser, J. Mol. Spectrosc. 204, 133 (2000).

${ }^{57}$ L. Bizzocchi, C. Degli Esposti, and P. Botschwina, J. Mol. Spectrosc. 225, 145 (2004).

${ }^{58}$ H.S.P. Müller, B.J. Drouin, and J.C. Pearson, Astron. Astrophys. 506, 1487 (2009).

${ }^{59}$ M. Niedenhoff, G. Winnewisser, K.M.T. Yamada, and S.P. Belov, J. Mol. Spectrosc. 169, 224 (1995).

${ }^{60}$ H.M. Pickett, J. Mol. Spectrosc. 148, 371 (1991).

${ }^{61}$ T. Hrenar, G. Rauhut and H.-J. Werner, J. Phys. Chem. A. 110, 2060 (2006).

62 J.F. Stanton, J. Chem. Phys. 139, 046102 (2013).

${ }^{63}$ P. Botschwina and R. Oswald, J. Chem. Phys. 129, 044305 (2008).

${ }^{64}$ P. Botschwina and R. Oswald, J. Mol. Spectrosc. 254, 47 (2009).

${ }^{65}$ P. Botschwina, C. Stein, P. Sebald, B. Schröder, and R. Oswald, Astrophys. J. 787, 72 (2014).

${ }^{66}$ Z. Kisiel, J. Mol. Spectrosc. 218, 58 (2003).

${ }^{67}$ M.E. Sanz, M.C. McCarthy, and P. Thaddeus, J. Chem. Phys. 122, 194319 (2005). 